Pacific Journal of Mathematics

CHARACTERIZATIONS OF CONDITIONAL EXPECTATION AS 


\title{
CHARACTERIZATIONS OF CONDITIONAL EXPECTATION AS A TRANSFORMATION ON FUNCTION SPACES
}

\author{
Shu-Teh Chen MoY
}

Introduction. Let $(\Omega, \mathcal{F}, \mu)$ be a probability space; that is, $\Omega$ is a collection of elements $u, v, w, \ldots ;$; a $\sigma$-algebra of subsets of $\Omega$; and $\mu$, a countably additive measure on $\mathcal{F}$ with $\mu(\Omega)=1$. Let $x$ be a function defined on $\Omega$, having values on the real line extended by the adjunction of $+\infty$ and $-\infty$. Let $x$ be measurable with respect to 7 . We say that the expectation of $x$ exists if one of the integrals

$$
\int x+d \mu, \int x^{-} d \mu
$$

is finite where $x^{+}, x^{-}$are the positive part and the negative part of $x$, respectively. The expectation of $x, \mathrm{E}\{x\}$, is then defined to be equal to the integral $\int x d \mu$. Let $\mathcal{F}_{1}$ be a $\sigma$-algebra of subsets of $\Omega$ with $\mathcal{F}_{1} \subset \mathcal{F}^{\prime}$. For every $A \in \mathcal{F}_{1}$, the equation

$$
\phi(A)=\int_{A} x d \mu
$$

defines a countably additive set function $\phi$ on $\mathcal{F}_{1}$ which is absolutely continuous with respect to the contraction of $\mu$ to $\mathcal{F}_{1}$. By a generalized form of the RadonNikodym theorem there is an extended real-valued function $y$ defined on $\Omega$ which is measurable with respect to $\mathcal{F}_{1}$ and satisfies the equation

$$
\phi(A)=\int_{A} y d \mu
$$

Received January 13, 1953. The work covered in this paper was done under a predoctoral fellowship tenure from the Atomic Energy Commission. The author feels greatly indebted to the encouraging inspiration and comments of Profess or J. L. Doob under whose direction this paper was prepared. It constitutes part of a doctoral thesis submitted to the University of Michigan.

Pacific J. Math. 4 (1954), 47-64 
for every $A \in \mathcal{F}_{1}[3]$. Such a function is unique within $\mu$-measure 0 and is defined to be the conditional expectation of $x$ relative to $\mathcal{F}_{1}$, denoted by $E\left\{x \mid \mathcal{F}_{1}\right\}$. The integral or expectation of $x, \mathrm{E}\{x\}$, is then the special case of conditional expectation of $x$ relative to $\mathcal{F}_{1}$, where

$$
\mathcal{F}_{1}=\{\Omega, \text { null set }\} \text {. }
$$

In the following we shall list some properties of conditional expectation [2, Ch. $1 ; 6$, Ch. 5$]: x, y, z, x_{n}, \ldots$ are extended real-valued, measurable functions whose expectations exist.

CE 1. If $\alpha$ is a finite real number, then

$$
E\left\{\alpha x \mid \mathfrak{F}_{1}\right\}=\alpha E\left\{x \mid \mathfrak{F}_{1}\right\}
$$

almost everywhere. If $x$ is nonnegative almost everywhere, then $(1)$ is true for either finite or infinite $\alpha$.

CE 2. If

$$
\mathrm{E}\{x\}>-\infty, \mathrm{E}\{y\}>-\infty,
$$

then

$$
\mathrm{E}\left\{x+y \mid \mathcal{F}_{1}\right\}=\mathrm{E}\left\{x \mid \mathcal{F}_{1}\right\}+\mathrm{E}\left\{y \mid \mathcal{F}_{1}\right\}
$$

almost everywhere.

CE 3. If $x \geq y$ almost everywhere, then

$$
\mathrm{E}\left\{x \mid \mathcal{F}_{1}\right\} \geq E\left\{y \mid \mathcal{F}_{1}\right\}
$$

almost everywhere.

CE 4. The relation

$$
\left|E\left\{x \mid F_{1}\right\}\right| \leq E\left\{|x| \mid F_{1}\right\}
$$

holds almost everywhere. Therefore, if $x$ is equal to a bounded function almost everywhere then $E\left\{x \mid \mathcal{F}_{1}\right\}$ is also.

CE 5. If $x$ is measurable with respect to $F_{1}$ and $x$ is equal to a bounded function almost everywhere, and $y$ has finite expectation, then 


$$
\mathrm{E}\left\{x y \mid \mathcal{F}_{1}\right\}=x \mathrm{E}\left\{y \mid \mathcal{F}_{1}\right\}
$$

almost everywhere. If $x, y$ are nonnegative, then (2) is true for any $x$ which is measurable with respect to $\mathcal{F}_{1}$.

CE 6. If

$$
\mathrm{E}\left\{x_{n}\right\}>-\infty
$$

for all $n$, and $x_{1} \leq x_{2} \leq \cdots$ almost everywhere, then $E\left\{x_{n} \mid F_{1}\right\}$ converges almost everywhere to $\mathrm{E}\left\{x \mid \mathcal{F}_{1}\right\}$, where $x=\lim _{n \rightarrow \infty} x_{n}$ almost everywhere.

CE 7. If $p \geq 1$, then

$$
\left|\mathrm{E}\left\{x \mid \mathfrak{F}_{1}\right\}\right|^{p} \leq \mathrm{E}\left\{\left.|x|^{p}\right|^{\mathcal{F}_{1}}\right\}
$$

almost everywhere. Therefore, if

$$
\mathrm{E}\left\{|x|^{p}\right\}<\infty,
$$

then

$$
\mathrm{E}\left\{\left|\mathrm{E}\left\{x \mid \mathcal{F}_{1}\right\}\right|^{p}\right\}<\infty
$$

and if

$$
\mathrm{E}\left\{\left|x_{n}\right|^{p}\right\}<\infty
$$

for every $n$ and

$$
\lim _{n \rightarrow \infty} \mathrm{E}\left\{\left|x_{n}-x\right|^{p}\right\}=0
$$

then

$$
\lim _{n \rightarrow \infty} E\left\{\left|E\left\{x_{n} \mid \mathcal{F}_{1}\right\}-E\left\{x \mid \mathcal{F}_{1}\right\}\right|^{p}\right\}=0
$$

In this paper we shall study the relation of the conditional expectation with a transformation $T$ on some spaces of measurable functions into themselves satisfying the conditions:

$$
T(x+y)=T x+T y,
$$




$$
T(x T y)=(T x) \cdot(T y) .
$$

Under the restrictions that the transform of a function which is equal to a bounded function almost everywhere is also equal to a bounded function almost everywhere, and that $T$ satisfies a certain continuity condition, we are able to identify such a transformation as the one which takes $x$ to $\mathrm{E}\left\{x g \mid \mathcal{F}_{T}\right\}$, where $g$ is a measurable function and $\mathcal{F}_{T}$ is a $\sigma$-algebra of subsets of $\Omega$ with $\mathcal{F}_{T} \subset \mathcal{F}_{\text {. }}$ This is an attempt to answer the search for an appropriate definition of average which would be desirable for the establishment of a mathematical theory of the dynamics of turbulence [5, 7]. In the past, J. Kampé De Fériet has studied the transformation on the collection of real functions which takes only a finite number of values [4]. Garret Birkhoff and John Sopka have studied the transformation on the space of continuous functions on a compact Hausdorff space $[1,8]$. Garrett Birkhoff also treated the subject from an abstract algebraic point of view [1]. Since the modern treatment of fluid dynamics theory is based on probability theory, it seems to the author that a probability solution is the natural one.

In the first section of this paper we shall consider the transformation on the space of nonnegative measurable functions into itself. In some respect it is analogous to the integration theory of nonnegative functions. In the second section we consider $T$ as a linear continuous transformation on $L_{p}$ into $L_{p}$. In the case of $L_{1}$ it is also proved, if $T 1=1$ and $\|T x\|_{1} \leq\|x\|_{1}$ where $\|\cdot\|_{1}$ denotes the $L_{1}$ norm, then $T x$ is the conditional expectation of $x$ relative to a $\sigma$-algebra of subsets.

1. Transformation on the space of nonnegative measurable functions. Let $\&$ be the collection of all nonnegative, extended real-valued functions on $\Omega$ which are measurable with respect to $f$. Elements of $\&$ are denoted by $x, y$, $z, \ldots$. Two functions are considered equal if they are equal almost everywhere. By $x=y$ or $x>y$ we mean that $x=y$ almost everywhere or $x>y$ almost everywhere, respectively. When we say that $x$ is bounded we mean that $x$ is equal to a bounded function almost everywhere. We shall use the symbol $x_{n} \rightarrow x$ to mean that

$$
\lim _{n \rightarrow \infty} x_{n}(w)=x(w)
$$

for almost all $w$ in $\Omega$. Addition and multiplication in $\&$ are ordinary pointwise addition and multiplication with the conventions that $\alpha+\infty=\infty$ for every nonnegative number $\alpha$, and $\alpha \cdot \infty=\infty$ if $\alpha>0, \alpha \cdot \infty=0$ if $\alpha=0$. Thus \& is closed under addition and multiplication. We shall use symbols $\alpha, \beta, \cdots$ to 
denote nonnegative real numbers. We shall use the same symbols to denote functions which take on a constant value $\alpha, \beta, \ldots$ almost everywhere.

We are considering a transformation $T$ on $\&$ into $\&$ satisfying the following conditions:

T1. a) $T(x+y)=T x+T y$ for every pair of elements $x, y$ in \&.

b) $T \alpha x=\alpha T x$ for every nonnegative number $\alpha$ and every $x$ in 8 .

T2. If $x$ is bounded then Tx is bounded.

T3. $T(x \cdot T y)=(T x) \cdot(T y)$ for every pair of elements $x, y$ in \&.

T4. If $\left\{x_{n}\right\}$ is a nondecreasing sequence of elements of \& for which $x_{n} \rightarrow x$, then $T x_{n} \rightarrow T x$.

If $T_{E}$ is the transformation which takes $x$ to $\mathrm{E}\left\{x \mid \mathcal{F}_{1}\right\}$, then by CE $3 T_{E}$ is a transformation on $\&$ into $\&$. CE 1 and CE 2 imply that $T_{E}$ satisfies the condition T1; CE 4 implies that $T_{E}$ satisfies the condition T2; CE 5 implies that $T_{E}$ satisfies the condition T3; and CE 6 implies that $T_{E}$ satisfies the condition T4. Therefore the transformation which takes $x$ to the conditional expectation of $x$ relative to a $\sigma$-algebra of subsets $\mathcal{F}_{1} \subset \mathcal{F}$ satisfies T1, T2, T3, and T4. It is easy to check that the transformation which takes $x$ to $\mathrm{E}\left\{x g \mid \mathcal{F}_{1}\right\}$, where $g$ is a nonnegative measurable function with $\mathrm{E}\left\{\mathrm{g} \mid \mathcal{F}_{1}\right\}$ bounded, also satisfies $\mathrm{T} 1, \mathrm{~T} 2, \mathrm{~T} 3$, and $\mathrm{T} 4$. We shall prove that the last example is actually the most general form of a transformation satisfying T1, T2, T3, and T4.

LEMMA 1.1. The inequality $x \geq y$ implies $T x \geq T y$.

Proof. If $y$ is finite valued almost everywhere, then

$$
T x=T(y+(x-y))=T x+T(x-y) \geq T y .
$$

If $y$ is not finite valued, let

$$
A=[w: y(w)=\infty]
$$

then $x(w)=\propto$ for $w \in A$. Let $x^{\prime}, y^{\prime}$ be defined as $x^{\prime}(w)=x(w)$ for $w \notin A$, $x^{\prime}(w)=0$ for $w \in A ; y^{\prime}(w)=y(w)$ for $w \notin A$, and $y^{\prime}(w)=0$ for $w \in A$. Then $T x^{\circ} \geq T y^{\prime}$. Let $z$ be a function defined as $z(w)=\infty$ if $w \in A, z(w)=0$ if $w \notin A$; then

$$
x=x^{\prime}+z, y=y^{\prime}+z,
$$

and 


$$
T x=T x^{\prime}+T z \geq T y^{\prime}+T z=T y \text {. }
$$

LEMMA 1.2. If $x_{n} \rightarrow x$, then $T x \leq \lim _{n} \inf T x_{n}$.

Proof. Let

$$
y_{n .}=\inf \left[x_{i}: i \geq n\right]
$$

then $y_{n} \leq x_{n}$ for every $n$ and $\left\{y_{n}\right\}$ is a nondecreasing sequence of elements for which $y_{n} \rightarrow x$. By T4, $T y_{n} \rightarrow T x$. But by Lemma 1.1, $T y_{n} \leq T x_{n}$ for every $n$; hence

$$
T x \leq \lim _{n} \inf T x_{n}
$$

LEMMA 1.3. If $x_{n} \longrightarrow x$ and $x_{n} \leq y$ for every $n$ where $y$ and Ty are finite valued, then $T x_{n} \rightarrow T x$.

Proof. By Lemma 1.2,

$$
T x \leq \lim _{n} \inf T x_{n}
$$

and

$$
T(y-x) \leq \lim _{n} \inf T\left(y-x_{n}\right)
$$

By Lemma 1.1, $T x_{n} \leq T y$ for every $n$ and $T x \leq T y$; hence the second inequality can be written as

$$
T y-T x \leq T y-\lim _{n} \sup T x_{n}
$$

that is,

$$
T x \geq \lim _{n} \sup T x_{n}
$$

Now we have

$$
\lim _{n} \sup T x_{n} \leq T x \leq \lim _{n} \inf T x_{n}
$$

hence

$$
T x_{n} \rightarrow T x
$$


LEMMA 1.4. Let $\mathcal{E}$ be the totality of elements $y$ of $\&$ for which $T(x y)=y T x$ for every $x \in \&$.

1. If $y_{1}, y_{2} \in \mathcal{E}$, then $y_{1}+y_{2}$ and $y_{1} \cdot y_{2} \in \mathcal{E}$; and if $y_{1} \leq y_{2}$ with $y_{2}$ bounded, then $y_{2}-y_{1} \in \mathcal{E}$.

2. If $\alpha \geq 0, y \in \varepsilon$, then $\alpha y \in \mathcal{E}$.

3. If $\left\{y_{n}\right\}$ is a nondecreasing sequence of elements with $y_{n} \in \mathcal{E}$ for every $n$ and $y_{n} \rightarrow y_{\text {, then }} y \in \mathcal{E}$.

4. If $\left\{y_{n}\right\}$ is a sequence of elements in $\mathcal{E}$ with $y_{n} \rightarrow y$ and there is a bounded function $z$ for which $y_{n} \leq z$ for every $n$, then $y \in \mathcal{E}$.

Proof. 1. We have

$$
T\left[x\left(y_{1}+y_{2}\right)\right]=T\left(x y_{1}\right)+T\left(x y_{2}\right)=y_{1} T x+y_{2} T x=\left(y_{1}+y_{2}\right) T x,
$$

and

$$
T\left(x y_{1} y_{2}\right)=y_{1} T\left(x y_{2}\right)=y_{1} y_{2} T x .
$$

If $y_{2}$ is bounded and $y_{1} \leq y_{2}$, then

$$
\begin{aligned}
y_{2} T x=T\left(x y_{2}\right)=T\left[x\left(y_{1}+y_{2}-y_{1}\right)\right] & =T\left(x y_{1}\right)+T\left[x\left(y_{2}-y_{1}\right)\right] \\
& =y_{1} T x+T\left[x\left(y_{2}-y_{1}\right)\right] .
\end{aligned}
$$

If $x$ is bounded, then $T x$ is bounded. We have

$$
y_{2} T x-y_{1} T x=\left(y_{2}-y_{1}\right) T x=T\left[x\left(y_{2}-y_{1}\right)\right] .
$$

The last equality is true for all bounded $x$, and therefore is true for all $x$.

2. Clearly

$$
T(x \alpha y)=\alpha T(x y)=\alpha y T x .
$$

3. We have $T\left(x y_{n}\right)=y_{n} T x$ for every $n$. Further, $T\left(x y_{n}\right)$ and $y_{n} T x$ are nondecreasing sequences with $T\left(x y_{n}\right) \rightarrow T(x y)$, by T4; and $y_{n} T x \rightarrow y T x$. Hence $T(x y)=y T x$.

4. We have $T\left(x y_{n}\right)=y_{n} T x$ for every $n$. If $x$ is bounded, then $x z$ is bounded and $x y_{n} \leq x z$ for each $n$. By Lemma 1.3, $T\left(x y_{n}\right) \rightarrow T(x y)$. On the other hand, we have $y_{n} T x \rightarrow y T x$. Hence $T(x y)=y T x$. The equality is true for all bounded 
$x$, and therefore is true for all $x$ by T4.

We remark that 1 . implies that any nonnegative polynomial $P(y)$, of a bounded $y \in \mathcal{E}$ (that is, $P(y(w)) \geq 0$ for all $w \in \Omega$ ) is also in $\varepsilon$.

The following lemma is obvious by $\mathrm{T} 3$.

Lemia 1.5. For each $x \in$ \&, $T x \in \mathcal{E}$.

LEMм A 1.6. Let $\mathcal{F}_{T}$ be the collection of all sets $E \in \mathcal{F}$ whose characteristic functions $\chi_{E}$ are in the $\mathcal{E}$ of Lemma 1.4. Then $\mathcal{F}_{T}$ is a $\sigma$-algebra of subsets of $\Omega$.

Proof. We shall establish:

1. Clearly, $1 \in \mathcal{E}$ for $1 T x=T(x \cdot 1)$; hence $\Omega \in \mathcal{F}_{T}$.

2. If $E_{1}, E_{2} \in \mathcal{F}_{T}$, then $E_{1} \cap E_{2} \in \mathcal{F}_{T}$ by Lemma 1.4 and the equation

$$
\chi_{E_{1}} \cap E_{2}=\chi_{E_{1}} \cdot \chi_{E_{2}}
$$

3. If $E_{1}, E_{2} \in \mathcal{F}_{T}$, then $E_{1}-E_{2} \in \mathcal{F}_{T}$ by the equality

$$
\chi_{E_{1}-E_{2}}=\chi_{E_{1}}-\chi_{E_{1}} \cap E_{2}
$$

and Lemma 1.4.

4. If $E_{1}, E_{2} \in \mathcal{F}_{T}$, then $E_{1} \cup E_{2} \in \mathcal{F}_{T}$ by Lemma 1.4 and the equality

$$
\chi_{E_{1} \cup E_{2}}=\chi_{E_{1}}+\chi_{E_{2}}-\chi_{E_{1} \cap E_{2}}
$$

By induction, for any finite number of sets $E_{1}, E_{2}, \ldots E_{n} \in \mathcal{F}_{T}$,

$$
\bigcup_{i=1}^{n} E_{i} \in \mathcal{F}_{T} \text {. }
$$

5. For a sequence of sets $E_{1}, E_{2}, \ldots, E_{n}, \ldots$ in $\mathcal{F}_{T}$.

$$
\bigcup_{n=1}^{\infty} E_{n} \in \mathcal{F}_{T} .
$$

For, by 4, 


$$
\chi_{\bigcup_{i=1}^{n} E_{i}} \in \mathcal{E}
$$

and

$$
\left\{X_{U_{i=1}^{n}} E_{i}\right\}
$$

is a nondecreasing sequence for which

$$
\chi_{U_{i=1}^{n} E_{i}} \rightarrow \chi_{U_{i=1}^{\infty} E_{i}}
$$

LEMMA 1.7. Let $M$ be the collection of all nonnegative functions which are measurable with respect to $\mathcal{F}_{T}$. Then $m \subset \mathcal{E}$.

Proof. Functions which are linear combinations with nonnegative coefficients of characteristic functions of sets in $\mathcal{F}_{T}$ are in $\mathcal{E}$. For any element of $m$ there is a nondecreasing sequence of such functions converging to it; therefore, by Lemma 1.4, it is in $\varepsilon$.

LеммA 1.8. Let $y \in \mathcal{E}$ and $y$ be bounded. Let $\mathcal{F}_{y}$ be the least $\sigma$-algebra with respect to which $y$ is measurable. Then $\mathfrak{F}_{y} \subset \mathcal{F}_{T}$, or, equivalently, $y \in m$.

Proof. Let $\Phi$ be a nonnegative continuous function on a finite interval containing the range of $y$. We want to prove $\Phi(y) \in \mathcal{E}$. We may assume that

$$
0<\alpha \leq \Phi(y) \leq \beta
$$

for $\Phi(y)+\alpha \in \mathcal{E}$ with $\alpha>0$ implies that $\Phi(y)^{\circ} \in \mathcal{E}$, by Lemma 1.4.

Since $y$ is bounded by the Weierstrass theorem there is a sequence $\left\{P_{n}(y)\right\}$ of polynomials such that $P_{n}(y)$ converges uniformly to $\Phi(y)$. We may assume $P_{n}(y(w)) \geq 0$ for all $w \in \Omega$; therefore, by Lemma 1.4, $P_{n}(y) \in \mathcal{E}$ for each $n$, and $\Phi(y) \in \mathcal{E}$.

For each $E \in \mathcal{F}_{y}$, there is a sequence $\left\{\Phi_{n}(y)\right\}$ of continuous functions of $y$ with $0 \leq \Phi_{n}(y(w)) \leq 1$ for each $n$ and $w$ for which

$$
\Phi_{n}(y) \rightarrow \chi_{E}
$$

Hence, again by Lemma 1.4, $\chi_{E} \in \mathcal{E}$; that is, $E \in \mathcal{F}_{T}$.

Lемма 1.9. For each $x \in$ e, $T x \in \mathbb{M}$. 
Proof. Let $\left\{x_{n}\right\}$ be a nondecreasing sequence of bounded functions for which $x_{n} \rightarrow x$. Then $\left\{T x_{n}\right\}$ is also a nondecreasing sequence of bounded functions, and $T x_{n} \rightarrow T x$. By Lemma 1.5 and Lemma 1.8, $T x_{n} \in M$ for every $n$; therefore $T x \in M$.

THEOREM 1.1. If $T$ is a transformation on the collection s of all nonnegative measurable functions on a probability space $(\Omega, \mathcal{F}, \mu)$ into itself satisfying $\mathrm{T} 1, \mathrm{~T} 2, \mathrm{~T} 3, \mathrm{~T} 4$, then $T$ is of the form

$$
T x=\mathrm{E}\left\{x g / \mathcal{F}_{T}\right\},
$$

where $\mathcal{F}_{T}$ is a $\sigma$-algebra of subsets of $\Omega$ with $\mathcal{F}_{T} \subset \mathcal{F}$ and $g$ is a nonnegative measurable function for which $\mathrm{E}\left\{\mathrm{g} \mid \mathrm{J}_{T}\right\}$ is bounded.

Proof. Consider the set function $\nu$ on $\mathcal{F}$ defined by

$$
\nu(A)=\int T \chi_{A} d \mu
$$

where $\chi_{A}$ is the characteristic function of $A$; then $\mathrm{T} 1, \mathrm{~T} 2, \mathrm{~T} 4$ imply that $\nu$ is a finite measure on $\mathcal{F}$. For a linear combination of characteristic functions with nonnegative coefficients

$$
\sum_{i=1}^{n} \alpha_{i} x_{A_{i}}
$$

we have

$$
\int T\left(\sum_{i=1}^{n} \alpha_{i} \chi_{A_{i}}\right) d \mu=\sum_{i=1}^{n} \alpha_{i} \int T \chi_{A_{i}} d \mu=\sum_{i=1}^{n} \alpha_{i} \nu\left(A_{i}\right)
$$

Hence for each $x \in £$,

$$
\int T x d \mu=\int x d \nu
$$

Since $\nu$ is absolutely continuous with respect to $\mu$, by the Radon-Nikodym theorem there is a nonnegative function $g$ for which

$$
\nu(A)=\int_{A} g d \mu
$$

for every $A \in \mathcal{F}$. Therefore for each $x \in \mathcal{D}$, 


$$
\int T x d \mu=\int x g d \mu
$$

For every $E \in \mathcal{F}_{T}$,

$$
\int_{E} T x d \mu=\int \chi_{E} T x d \mu=\int T\left(x \chi_{E}\right) d \mu=\int x \chi_{E} g d \mu=\int_{E} x g d \mu .
$$

The previous equality and the fact that $T x$ is measurable with respect to $\mathcal{F}_{T}$ (Lemma 1.9) imply that

$$
T x=E\left\{x g \mid \mathcal{F}_{T}\right\}
$$

In particular, $\mathrm{Tl}=\mathrm{E}\left\{\mathrm{g} \mid \mathcal{F}_{T}\right\}$; hence $\mathrm{E}\left\{\mathrm{g} \mid \mathrm{F}_{T}\right\}$ is bounded.

REMARK. The representation $T x=E\left\{g x \mid \mathcal{F}_{T}\right\}$ is not unique. For example, if $g(w)=0$ for $w \in E$, where $E \in \mathcal{F}_{T}$ and $\mu(E)>0$, and we let

$$
\mathfrak{F}^{\circ}=\left[E \cup F: F \in \mathcal{F}_{T}, F \cap E=\phi\right] \cup\left[F: F \in \mathcal{F}_{T}, F \cap E=\phi\right],
$$

then $E\left\{x g \mid \mathcal{F}_{T}\right\}=E\left\{x g \mid \mathcal{F}^{\bullet}\right\}$ for every $x$ in $\$$.

COROLLARY 1.1. If the collection of nonnegative constant functions is invariant under $T$ satisfying $\mathrm{T} 1, \mathrm{~T} 2, \mathrm{~T} 3, \mathrm{~T} 4$, then, except for the trivial case $T x=0$ for all $x$, the range of $T$ is $m$, and $g$ of Theorem 1.1 satisfies

$$
E\left\{g \mid \mathfrak{F}_{T}\right\}=\alpha,
$$

where $\alpha=\mathrm{T} 1 \neq 0$. In particular, if $\mathrm{Tl}=1$, then $T$ is a projection of \& on $\mathrm{m}$.

Proof. Tl must not be 0 . For if $\mathrm{T} 1=0$ then

$$
\int g d \mu=\int \mathrm{T} 1 d \mu=0 .
$$

Therefore $g=0$, and $T x=0$ for every $x$. For each $y \in m$,

$$
T y=\mathrm{E}\left\{y g \mid \mathfrak{F}_{T}\right\}=y \mathrm{E}\left\{g \mid \mathfrak{F}_{T}\right\}=\alpha y ;
$$

therefore

$$
T \frac{1}{\alpha} y=y .
$$

This fact together with Lemma 1.9 implies that $m$ is the range of $T$. If $\mathrm{T} l=1$, then 


$$
T^{2} x=T(1 \cdot T x)=T x \mathrm{~T} 1=T x ;
$$

that is, $T^{2}=T$. Hence $T$ is a projection in this case.

2. Transformation on the space $L_{p}$. Let $L_{p}, p \geq 1$, be the usual space of all real $p$ th power integrable functions on the probability space $(\Omega, \mathcal{F}, \mu)$. If the transformation discussed in the previous section takes functions in $L_{p}$ into functions in $L_{p}$, then it can be extended to be a linear transformation on $L_{p}$ into $L_{p}$ by defining $T x=T x^{+}-T x^{-}$, where $x^{+}, x^{-}$are the positive and negative parts of $x$, respectively; therefore we still have the same representation

$$
T x=E\left\{x g \mid \mathcal{F}_{T}\right\}
$$

for every $x$ in $L_{p}$ with a nonnegative function $g$ for which $\mathrm{F}\left\{g \mid \mathcal{F}_{T}\right\}$ is bounded. The restriction that $T$ transforms nonnegative functions into nonnegative functions is the same as that $T$ be order preserving; that is, if $x \geq y$ then $T x \geq T y$. In this section we shall consider a transformation on $L_{p}$ into $L_{p}$ similar to the previous one, but with no restriction that the order is to be preserved. We shall employ the usual norm topology in $L_{p}$ and assume the transformation to be continuous in norm topology. We are able to prove with essentially the same argument as in the previous section that the same representation

$$
T x=\mathrm{E}\left\{x g \mid \mathcal{F}_{T}\right\}
$$

is to be arrived at, but with a function $g$ which is no longer nonnegative.

We shall state the assumptions precisely. In the following, elements of $L_{p}$ are denoted by $x, y, z, \ldots$. As before, two functions are considered equal if they are equal almost everywhere. A function is said to be bounded if it is equal to a bounded function almost everywhere. The symbols $\alpha, \beta, \ldots$ are to denote both real constants and functions which take a constant value almost everywhere. For a sequence of functions $\left\{x_{n}\right\}$ we shall use the expression " $x_{n} \rightarrow x$ in $L_{p}$ " to denote

$$
\lim _{n \rightarrow \infty} \int\left|x_{n}-x\right|^{p} d \mu=0
$$

$T$ is to be a transformation on $L_{p}$ into $L_{p}$ satisfying the following conditions:

$\mathrm{T}^{\prime} 1 . T$ is linear; that is,

$$
T(\alpha x+\beta y)=\alpha T x+\beta T y .
$$

$\mathrm{T}^{\prime 2}$. If $x$ is bounded, then $T x$ is bounded. 
T'3. $T(x T y)=(T x) \cdot(T y)$ for every pair $x, y$ of bounded elements of $L_{p}$.

$\mathrm{T}^{\prime} 4$. T is continuous; that is, if $x_{n} \rightarrow x$ in $L_{p}$ then $T x_{n} \rightarrow T x$ in $L_{p}$.

From the properties of the conditional expectation $\mathrm{CE} 1, \mathrm{CE} 2, \mathrm{CE} 5, \mathrm{CE} 6$, and $\mathrm{CE} 7$, we see that the transformation which takes $x$ to $\mathrm{E}\left[x \mid \mathcal{F}_{1}\right]$ satisfies the foregoing conditions.

LEMMA 2.1. Let $\mathcal{E}$ be the totality of elements $y$ of $L_{p}$ for which $T(x y)=y T x$ holds for every bounded $x$ in $L_{p}$. Then $\mathcal{E}$ is a closed linear subspace of $L_{p}$. Moreover, if $y_{1}, y_{2} \in \mathcal{E}$ and $y_{1}, y_{2}$ are bounded, then $y_{1} \cdot y_{2} \in \mathcal{E}$; therefore every polynomial of a bounded function in $\mathcal{E}$ is also in $\mathcal{E}$.

Proof. The proof that $\mathcal{E}$ is a linear set and closed under multiplication of bounded functions is similar to that of Lemma 1.4. To prove that it is a closed subset of $L_{p}$, let $y_{n} \rightarrow y$ in $L_{p}$ and $y_{n} \in \mathcal{E}$ for every $n$; then $x y_{n} \rightarrow x y$ in $L_{p}$ for every bounded $x$, and hence $T\left(x y_{n}\right) \rightarrow T(x y)$ in $L_{p}$, by T'4. On the other hand, if $x$ is bounded, then $T x$ is also bounded by $\mathrm{T}^{\prime} 2$; hence

$$
y_{n} T x \rightarrow y T x
$$

in $L_{p}$. Since

$$
y_{n} T x=T x y_{n}
$$

for every $n$, we have $y T x=T x y$; that is, $y \in \mathcal{E}$. Hence $\mathcal{E}$ is a closed subset of $L_{p}$.

Lemma 2.2. For each $x \in L_{p}, T x \in \mathcal{E}$.

Proof. By $\mathrm{T}^{\prime} 3$, if $x$ is bounded then $T x \in \mathcal{E}$. If $x$ is not bounded, there is a sequence $\left\{x_{n}\right\}$ of bounded functions for which $x_{n} \rightarrow x$ in $L_{p}$; then $T x_{n} \rightarrow T x$ in $L_{p}$, by $\mathrm{T}^{\prime} 4$. Now $T x_{n} \in \mathcal{E}$ for each $n$, and the fact that $\mathcal{E}$ is closed (Lemma 2.1 ), imply that $T x \in \mathcal{E}$.

LEMMA 2.3. Let $\mathcal{F}_{T}$ be the collection of all sets $E \in \mathcal{F}$ whose characteristic functions $\chi_{E}$ are in $\mathcal{E}$; then $\mathcal{J}_{T}$ is a $\sigma$-algebra of subsets of $\Omega$.

The proof is the same as that of Lemma 1.6 except that we have to use the fact that $\mathcal{E}$ is a closed subset of $L_{p}$ and

$$
\chi_{U_{i=1}^{n} E_{i}} \rightarrow \chi_{U_{i=1}^{\infty} E_{i}}
$$


in $L_{p}$ to prove that $\mathcal{F}_{T}$ is closed under countable unions.

LeмmA 2.4. Let $m$ be the totality of elements of $L_{p}$ which are measurable with respect to the $\mathcal{F}_{T}$ of Lemma 2.3 , then $m \subset \mathcal{E}$.

Proof. Linear combinations of characteristic functions of sets of $\mathcal{F}_{T}$ are in $\varepsilon$. The facts that the totality of such functions is dense in $m$, and that $\varepsilon$ is a closed subset of $L_{p}$, imply that $m \subset \varepsilon$.

Lemma 2.5. Let $y \in \mathcal{E}$ and $y$ be bounded. Let $\mathcal{F}_{y}$ be the smallest $\sigma$-algebra of subsets of $\Omega$ with respect to which $y$ is measurable. Then ${ }^{\mathcal{F}_{y}} \subset \mathcal{F}_{T}$ or, equivalently, $y \in M$.

Proof. Let $\Phi$ be a continuous real function on a finite interval containing the range of $y$. Since $y$ is bounded, by the Weierstrass theorem there is a sequence $\left\{P_{n}(y)\right\}$ of polynomials of $y$ for which $P_{n}[y(w)]$ converges to $\Phi[y(w)]$ uniformly in $w$. Hence $P_{n}(y) \rightarrow \Phi(y)$ in $L_{p}$; therefore, by Lemma 2.1, $P_{n}(y) \in \mathcal{E}$ for every $n$, and $\Phi(y) \in \mathcal{E}$.

For each $E \in \mathcal{F}_{y}$, there is a sequence $\left\{\Phi_{n}(y)\right\}$ of continuous functions of $y$ for which $\Phi_{n}(y) \rightarrow \chi_{E}$ in $L_{p}$, where $\chi_{E}$ is the characteristic function of $E$. Hence, again by Lemma 2.1, $\chi_{E} \in \mathcal{E}$; that is, $E \in \mathcal{F}_{T}$.

Lemma 2.6. For each $x \in L_{p}, T x \in M$.

Proof. For each $x \in L_{p}$ there is a sequence $\left\{x_{n}\right\}$ of bounded functions in $L_{p}$ for which $x_{n} \rightarrow x$ in $L_{p}$. Hence $T x_{n} \rightarrow T x$ in $L_{p}$ by $\mathrm{T}^{\prime} 4$. By $\mathrm{T}^{\prime} 2, T x_{n}$ is bounded for every $n$. By Lemma 2.2 and Lemma 2.5, $T x_{n} \in M$ for every $n$. Since $m$ is a closed subset of $L_{p}, T x \in M$.

THEOREM 2.1. If $T$ is a transformation of $L_{p}$ into $L_{p}$ satisfying $\mathrm{T}^{\prime} 1, \mathrm{~T}^{\prime} 2$, $\mathrm{T}^{\prime} 3, \mathrm{~T}^{\prime} 4$, then $T$ is of the form

$$
T x=\mathrm{E}\left\{x g \mid \mathfrak{F}_{T}\right\},
$$

where $\mathcal{F}_{T}$ is a $\sigma$-algebra of subsets of $S_{2}$ with $\mathcal{F}_{T} \subset \mathcal{F}$ and $g \in L_{q}$, where $1 / p+1 / q=1$, for which $\mathrm{E}\left\{\mathrm{g} \mid \mathrm{J}_{T}\right\}$ is bounded (in the case of $p=1$, then $g$ is a bounded function).

Proof. We consider the function $\ell(x)$ defined on $L_{p}$ by

$$
\ell(x)=\int T x d \mu
$$


$T^{\prime} 1$ implies that $l$ is linear. Also, $l$ is continuous, for if $x_{n} \rightarrow x$ in $L_{p}$ then $T x_{n} \rightarrow T x$ in $L_{p}$, which implies $T x_{n} \rightarrow T x$ in $L_{1}$; therefore

$$
\ell\left(x_{n}\right)=\int T x_{n} d \mu \rightarrow \int T x d \mu=\ell(x)
$$

Now $\ell$ can be expressed as

$$
\ell(x)=\int x g d \mu
$$

where $g \in L_{q}$ with $1 / p+1 / q=1$. (In the case $p=1, g$ is bounded almost everywhere.) Hence,

$$
\int T x d \mu=\int x g d \mu
$$

for every $x \in L_{p}$. The same argument as in the proof of Theorem 1.1 shows that

$$
T x=E\left\{x g \mid \mathcal{F}_{T}\right\},
$$

and that $E\left\{g \mid \mathcal{F}_{T}\right\}$ is bounded.

THEOREM 2.2. If $p=1$, and $T$ satisfies the further conditions that

$$
\mathrm{Tl}=1 \text { and }\|T x\|_{1} \leq\|x\|_{1}
$$

for every $x \in L_{1}$, where

$$
\|x\|_{1}=\int|x| d \mu
$$

then

$$
T x=\mathrm{E}\left\{x \mid \mathfrak{F}_{T}\right\}
$$

for every $x$.

Proof. Consider the set function $v$ defined on $F$

$$
\nu(E)=\ell\left(\chi_{E}\right)=\int T \chi_{E} d \mu,
$$

where $\chi_{E}$ is the characteristic function of $E$, and $\ell$ is the linear functional defined in the proof of Theorem 2.1. Since $\ell$ is continuous, $\nu$ is a completely additive set function. $\mathrm{T} l=1$ implies that $\nu(\Omega)=1$. For any set $E \in \mathcal{F}$, 


$$
\begin{aligned}
|\nu(E)|=\left|\int T \chi_{E} d \mu\right| \leq \int\left|T \chi_{E}\right| d \mu=\left\|T \chi_{E}\right\|_{1} \\
\\
\quad \leq\left\|\chi_{E}\right\|_{1}=\int \chi_{E} d \mu=\mu(E) .
\end{aligned}
$$

Now we want to show that $\nu(E)=\mu(E)$ for every $E \in \mathcal{F}$. First we shall prove

$$
|\nu(E)|=\mu(E) \text {. }
$$

Suppose

$$
|\nu(E)|<\mu(E)
$$

for a certain $E$; then

$$
1=\mu(\Omega)=\mu(E)+\mu(\Omega-E)>|\nu(E)|+|\nu(\Omega-E)| \geq \nu(\Omega)=1 \text {. }
$$

This is a contradiction. Hence

$$
|\nu(E)|=\mu(E)
$$

Now for any $E$, we have either

$$
\nu(E)=\mu(E) \text { or } \quad \nu(E)=-\mu(E) \text {. }
$$

Suppose

$$
\nu(E)=-\mu(E)
$$

then

$$
\nu(\Omega-E)=1+\mu(E)
$$

This is possible only when $\mu(E)=0$. Therefore

$$
\nu(E)=\mu(E)
$$

for every $E \in \mathcal{F}$.

The fact that $\nu \equiv \mu$ implies that the $g$ in Theorem 2.1 is equal to 1 almost everywhere, for

$$
\nu(E)=\ell\left(\chi_{E}\right)=\int_{E} g d \mu
$$


Hence the theorem is proved.

\section{REFERENCES}

1. Garrett Birkhoff, Moyennes des fonctions bornées, Congrès d'Algèbre et Théorie des Nombres, Paris, 1949, pp. $143-154$.

2. J. L. Doob, Stochastic processes (forthcoming book).

3. P. R. Halmos, Measure theory, New York, 1950.

4. J. Kampé De Fériet, Sur un problems d'algèbre abstrait posé par la definition de la moyenne dans la theorie de la turbulence. Ann. Soc. Sci. Bruxelles, 1, 63 (1949), 156172.

5.

Introduction to the statistical theory of turbulence, correlation and spectrum. Lecture Series No. 8, prepared by S. I. Pai, The Institute of Fluid Dynamics and Applied Mathematics, University of Maryland, 1950-51.

6. A. N. Kolmogorov, Foundations of the theory of probability, translated by N. Morrison, 1950.

7. Osborne, Reynolds, On the dynamical theory of incompressible viscous fluids, Philos. Trans. Roy. Soc. A, 136 (1895), $123-164$.

8. John Sopka, On the characterization of Reynolds operators on the algebra of all continuous functions on a compact Hausdorff space, Harvard thesis, June, 1950.

UNIVERSITY OF MICHIGAN

UNIVERSITY OF ILLINOIS 



\title{
PACIFIC JOURNAL OF MATHEMATICS
}

\section{EDITORS}

\author{
M. M. SCHIFFER* \\ Stanf ord University \\ Stanf ord, California \\ E. HewitT \\ University of Washington \\ Seattle 5, Washington
}

\author{
P. P. DILWCR TH \\ California Institute of Technology \\ Pasadena 4, California \\ E. F. BECKENBACH** \\ University of California \\ Los Angeles 24, California
}

\section{ASSOCIATE EDITORS}

$\begin{array}{ll}\text { H. BUSEMANN } & \text { P. R. HALMOS } \\ \text { HERDERT FF.DERER } & \text { HEINZ HOPF } \\ \text { MARSH ALLL IHALL } & \text { R. D. JAMES }\end{array}$

MARSH AL.L II ALL

\author{
R. D. JAMES
}
BORGE JESSEN
PAUL LÉVY
GEORGE PÓLYA

\author{
J. J. STOKER \\ E. G. STRAUS
}
KÖSAKU YOSIDA

\section{SPONSORS}

UNIVERSITY OF BRITISH COLUMBIA CAI IFORNIA ENSTITUTE OF TECHNOLOGY UNIVERSITY OF CALIFORNIA, BERKELEY UNIVERSITY OF CALIFORNIA, DAVIS UNIVERSITY OF CALIFORNIA, LOS ANGELES UNIVERSITY OF CALIFORNI A, SANTA BARBARA UNIVERSITY OF NEVADA OREGON STATE COLLEGE UNIVERSITY OF OREGON
UNIVERSITY OF SOU THERN CALIFORNIA STANFORD RESE.ARCH INSTITUTE STANFORD UNIVERSITY WASHING TON STATE COLLEGE UNIVERSITY OF WASHINGTON

AMERICAN MATHEMATICAL SOCIETY HUGHES AIRCRAFT COMPANY SHELL DEVELOPMENT COMPANY

* To be succeeded in 1955, by H.L. Royden, Stanford University, Stanford, California.

${ }^{* *}$ To be succeeded in 1955, by E.G. Straus, University of California, Los Angeles 24, Calif.

\author{
Vari-Type Composition by \\ Elaine Barth
}

Printed in the United States of America by

Edwards Brothers, Inc., Ann Arbor, Michigan

UNIVERSITY OF CALIFORNIA PRESS * BERKELEY AND LOS ANGELES COPYRIGHT 1954 BY PACIFIC JOURNAL OF MATHEMATICS 


\section{Pacific Journal of Mathematics}

\section{Vol. 4, No. 1 \\ May, 1954}

Hugh D. Brunk, On the growth of functions having poles or zeros on the positive real axis ................................. 1

J. Copping, Application of a theorem of Pólya to the solution of an infinite

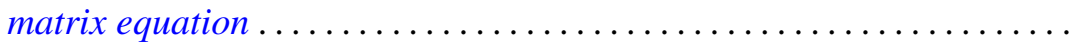

James Richard Jackson, On the existence problem of linear programming ................................... 29

Victor Klee, Invariant extension of linear functionals ............... 37

Shu-Teh Chen Moy, Characterizations of conditional expectation as a transformation on function spaces ..................... 47

Hukukane Nikaidô, On von Neumann's minimax theorem .............. 65

Gordon Marshall Petersen, Methods of summation .................. 73

G. Power, Some perturbed electrostatic fields .................. 79

Murray Harold Protter, The two noncharacteristic problem with data partly

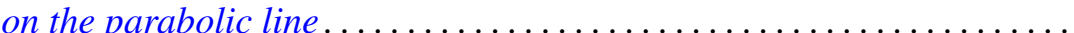

S. E. Rauch, Mapping properties of Cesàro sums of order two of the geometric series........................................... 109

Gerson B. Robison, Invariant integrals over a class of Banach spaces . . . . 123

Richard Steven Varga, Eigenvalues of circulant matrices .............. 151 